\title{
Identification of a Mechanism Underlying Regulation of the Anti-Angiogenic Forkhead Transcription Factor FoxO1 in Cultured Endothelial Cells and Ischemic Muscle
}

\author{
Malgorzata Milkiewicz, ${ }^{*}$ Emilie Roudier, ${ }^{\dagger}$ \\ Jennifer L. Doyle, ${ }^{\dagger}$ Anastassia Trifonova, ${ }^{\dagger}$ \\ Olivier Birot, ${ }^{\dagger}$ and Tara L. Haas ${ }^{\dagger}$
}

Chronic limb ischemia, a complication commonly observed in conjunction with cardiovascular disease, is characterized by insufficient neovascularization despite the up-regulation of pro-angiogenic mediators. One hypothesis is that ischemia induces inhibitory signals that circumvent the normal capillary growth response. FoxO transcription factors exert anti-proliferative and pro-apoptotic effects on many cell types. We studied the regulation of FoxO1 protein in ischemic rat skeletal muscle following iliac artery ligation and in cultured endothelial cells. We found that FoxO1 expression was increased in capillaries within ischemic muscles compared with those from rats that underwent a sham operation. This finding correlated with increased expression of $\mathrm{p2}^{\mathrm{Kip} 1}$ and reduced expression of Cyclin D1. Phosphorylated Akt was reduced concurrently with the increase in FoxO1 protein. In skeletal muscle endothelial cells, nutrient stress as well as lack of shear stress stabilized FoxO1 protein, whereas shear stress induced FoxO1 degradation. Endogenous FoxO1 co-precipitated with the E3 ubiquitin ligase murine double minute-2 (Mdm2) in endothelial cells, and this interaction varied in direct relation to the extent of Akt and Mdm2 phosphorylation. Moreover, ischemic muscles had a decreased level of Mdm2 phosphorylation and a reduced interaction between $\mathrm{Mdm} 2$ and FoxO1. Our results provide novel evidence that the Akt-Mdm2 pathway acts to regulate endothelial cell FoxO1 expression and illustrate a potential mechanism underlying the pathophysiological up-regulation of

FoxO1 under ischemic conditions. (Am J Pathol 2011, 178:935-944; DOI: 10.1016/j.ajpath.2010.10.042)

Successful reperfusion of the chronically ischemic limb depends both on re-establishment of blood flow via atherosclerotic feeding arteries and restoration of functioning microvessels through angiogenesis. Compensatory tissue neovascularization is therefore an important adaptive response to restore oxygen homeostasis in ischemic tissue. Unfortunately, the physiologic mechanisms that control microvascular growth and function ultimately fail within ischemic limbs. Although efforts have been made to deliver key mediators of hypoxia-induced angiogenesis such as vascular endothelial growth factor-A (VEGF-A) and hypoxiainducible factor- $1 \alpha(\mathrm{HIF}-1 \alpha),{ }^{1,2}$ clinical trials with pro-angiogenic agents have revealed little to no practical utility in patients who have chronic limb ischemia. ${ }^{3-6}$ These findings suggest a lack of responsiveness of endothelial cells to the growth factors or an elevated level of antiangiogenic signals that might circumvent the actions of angiogenic factors, but underlying mechanisms remain unknown. Thus it is crucial to better understand how anti-angiogenic signaling pathways may be induced by the ischemic environment.

An increasing body of evidence supports essential roles of the forkhead transcription factors (FOX) in vascular development and maturation. The FOX family is distinguished by a conserved 100 amino acid domain called the "forkhead box." The $O$ subgroup of this family (FoxO) comprises four members: FoxO1, FoxO3a, and FoxO4, which are widely expressed, and FoxO6, which is restricted to the brain. ${ }^{7-9}$ In response to a variety of metabolic, oxidative, and mechanical stresses, FoxO

Supported by grants from the Heart and Stroke Foundation of Canada (Grant in Aid NA6089 to T.L.H.) and the Polish Ministry of Science and Higher Education (N N401 051833 to M.M.).

Accepted for publication October 21, 2010.

M.M. and E.R. contributed equally to the study.

Address reprint requests to Tara L. Haas, Rm 341 Farquharson Bldg, York University, 4700 Keele St, Toronto, ON, Canada M3J 1P3. E-mail: thaas@yorku.ca. 
transcription factors promote cell cycle arrest, repair of damaged DNA, gluconeogenesis, apoptosis, and detoxification of reactive oxygen species. ${ }^{10-12}$ Of the three highly related members of the FoxO family, only the loss of FoxO1 causes embryonic lethality due to severe defects in vascular development. ${ }^{13,14}$

FoxO transcription factors play substantive roles in vascular homeostasis by modulating several downstream genes important for vessel formation and maturation. Angiopoietin-1, which promotes vascular stability, inhibits the expression of its antagonist angiopoietin-2 through activation of the FoxO pathway. ${ }^{15}$ FoxO1 and FoxO3a may elicit anti-angiogenic effects in part by transcriptional repression of endothelial nitric oxide synthase (eNOS) in endothelial cells. ${ }^{16}$ Members of the FoxO family are known to induce both cell cycle arrest via the enhanced transcription of cell cycle inhibitors such as p27 ${ }^{\text {Kip }}$ and p21 and apoptosis through synthesis of bim, a pro-apoptotic $B \mathrm{Cl}-2$ family member. ${ }^{17}$ Conversely, VEGF-induced survival and proliferation of endothelial cells is associated with the repression of FoxO1 transcriptional activity and down-regulation of the FoxO1 target gene p27 $7^{\mathrm{Kip}} .^{18}$

The FoxO protein family is regulated primarily by posttranslational modifications, including phosphorylation, acetylation, mono-ubiquitination, and polyubiquitination. ${ }^{8,9}$ These modifications control FoxO protein levels and subcellular localization, as well as the efficacy of DNA-binding and transcriptional activity. FoxO factors are regulated negatively by the serine/threonine protein kinase Akt. Dephosphorylated FoxO1 localizes to the nucleus and exhibits transcriptional activity, whereas Aktdependent phosphorylation of FoxO1 protein causes its nuclear exportation and promotes its proteasomal degradation. ${ }^{10,19}$ Earlier work identified Skp2-dependent ubiquitination of FoxO1 subsequent to serine 256 phosphorylation. ${ }^{20}$ Two recent studies provided evidence that murine double minute-2 (Mdm2), an E3 ubiquitin ligase itself activated by Akt-mediated phosphorylation, ${ }^{21}$ also regulates FoxO protein levels. ${ }^{22,23}$

FoxO1 expression is suppressed by Akt-dependent phosphorylation in cells stimulated by growth factors or insulin or in endothelial cells subjected to sustained fluid shear stimulation. ${ }^{10,24-26}$ Considering that ischemia conversely generates an environment in which cellular activation by these factors is diminished, we hypothesized that chronic ischemia would reduce Akt signaling, thus promoting accumulation of FoxO1 and resulting in expression of factors that may override or reduce the angiogenic response.

\section{Materials and Methods}

\section{Ethical Approval}

Animal studies were approved by the York University Committee on Animal Care and performed in accordance with Animal Care Procedures at York University and the American Physiological Society's Guiding Principles in the Care and Use of Animals.

\section{Animal Protocol}

Male Sprague-Dawley rats (200 to $250 \mathrm{~g}$, Charles River Laboratories, Saint-Constant, QC, Canada) were used for all experiments. Rats were subject to hind limb ischemia for $4(n=4), 7(n=4)$, or 14 days $(n=4)$. Limitation of blood flow was induced by unilateral ligation of the right common iliac artery performed about $0.5 \mathrm{~cm}$ below the aortic bifurcation. Surgical procedures were carried out under anesthesia (intraperitoneal injection of ketamine, $80 \mathrm{mg} / \mathrm{kg}$, and xylazine, $10 \mathrm{mg} / \mathrm{kg}$ ) as previously described. ${ }^{27}$ Rats that underwent a sham operation $(n=8)$ were used as controls. At the end of each experiment, rats were anesthetized and the right extensor digitorum longus (EDL) and the tibialis anterior (TA) muscles were removed, weighed, and frozen either in liquid nitrogen or in cooled isopentane for histochemistry samples. Animals were euthanized by exsanguination.

\section{Muscle Histology and Capillary to Fiber Ratio}

Cryosections of TA muscle $(10 \mu \mathrm{m})$ were fixed in 3.7\% formaldehyde and stained with toluidine blue $(10 \mathrm{mg} / \mathrm{ml}$ toluidine blue in $70 \%$ ethanol, diluted $1: 10$ in $1 \% \mathrm{NaCl}$ ) for 20 minutes. Sections were rinsed with PBS and mounted with AquaPerm media (Fisher Thermoscientific, Whitby, ON, Canada). Capillary to muscle fiber ratios were determined as an indicator of angiogenesis. Cryosections of TA muscle $(10 \mu \mathrm{m})$ were fixed in cold acetone and stained with isolectin (fluorescein isothiocyanate-conjugated Griffonia simplicifolia I; Vector Laboratories, Burlington, ON, Canada) diluted in PBS (1:100) for 30 minutes. In each case, sections were viewed with an Olympus microscope $(\times 20$ objective) and images were captured using a digital color charge coupled device camera (Hitachi, Tokyo, Japan). Capillary and muscle fiber counts were averaged from five independent fields of view per rat (sampling from equivalent oxidative and glycolytic regions of the muscle in each condition).

\section{Extraction and Western Blotting of Skeletal Muscle Samples}

Proteins were extracted from 20 to $40 \mathrm{mg}$ of frozen muscles using a radioimmunoprecipitation assay lysis buffer containing $1 \mathrm{mg} / \mathrm{ml}$ phenylmethylsulfonyl fluoride, 1 $\mathrm{mmol} / \mathrm{L} \mathrm{Na}_{3} \mathrm{VO}_{4}, 1 \mathrm{mmol} / \mathrm{L} \mathrm{NaF}$ (Sigma-Aldrich, Montreal, $\mathrm{QC}$, Canada), and $1 \mathrm{X}$ protease inhibitor cocktail (Complete mini; Roche Diagnostics, Laval, QC, Canada). Muscle lysis was performed using a Retsch MM400 tissue lyser (Retsch $\mathrm{GmbH}$, Haan, Germany). Proteins were quantified by bicinchoninic acid assay (Pierce, Fisher Thermoscientific). Samples were separated on SDS-polyacrylamide gel electrophoresis under reducing conditions and blotted onto a polyvinylidene difluoride membrane (Immobilon P; Millipore Corporation, Billerica, MA). Forty (40) $\mu \mathrm{g}$ of total protein were loaded, except for FoxO1 and phosphoserine 256 FoxO1 detection, for which $80 \mu \mathrm{g}$ total protein were required. After blocking with $5 \%$ fat-free milk, membranes were probed overnight at $4^{\circ} \mathrm{C}$ using antibodies from Cell Signaling Technology 
(New England Biolabs, Pickering, ON, Canada) against FoxO1 (\#2880), phosphoserine 256 FoxO1 (\#9461), Akt (\#9272), phosphoserine 473 Akt (\#4058), phospho-ERK (\#9101), ERK (\#9102), phospho-p38 (\#9212), p38 (\#9211), phospho-Mdm2 (\#3521), $\beta$-actin (\#4967), $\alpha \beta$ tubulin (\#2148), eNOS antibody (\#9586), or cleaved caspase3 (\#9664). Alternatively, blots were probed with HIF1 $\alpha$ (NB100-105, Novus Biologicals, Littleton, CO), VEGF-A (sc152; Santa Cruz Biotechnology, Santa Cruz, CA) or Mdm2 (Clone 2A10; kindly provided by Dr. M.E. Perry, National Cancer Institute, Frederick, MD). Membranes were incubated with donkey anti-rabbit horseradish peroxidase secondary antibody (GE Healthcare BioSciences, Inc., Baie d'Urfe, QC, Canada or Dako Canada, Mississauga, ON, Canada). Bound antibodies were detected using Super West Pico (Pierce, Fisher Thermoscientific) or Immobilon Western ECL (Millipore Corporation) as per manufacturer's instructions and imaged using autoradiography film (Hyperfilm; GE Healthcare Bio-Sciences, Inc.) or using an imaging station (Kodak 4000MM Pro; Carestream Molecular Imaging, Woodbridge, CT). Bands were quantified using FluorChem software (Alpha Innotech, Cell Biosciences, San Leandro, CA).

\section{RNA Extraction and Real-Time PCR Analysis}

A small amount of frozen skeletal muscle $(<10 \mathrm{mg}$ ) was homogenized in $300 \mu \mathrm{l}$ RLT buffer (Qiagen Inc. Canada, Mississauga, ON, Canada) using a Retsch MM400 tissue lyser (Retsch $\mathrm{GmbH}$ ). Total RNA was purified using the RNeasy Fibrous Tissue Mini Kit (P/N 74704; Qiagen Inc. Canada). Following deoxyribonuclease treatment, RNA was reverse transcribed using MMLV reverse transcriptase (Applied Biosystems Canada, Streetsville, ON, Canada). cDNA samples were analyzed by quantitative PCR using PCR supermix (P/N 11743, Invitrogen Canada, Burlington, ON, Canada) and Taqman probes (Applied Biosystems Canada) as specified: glyceraldehyde-3-phosphate dehydrogenase (Rn99999916_s1), hypoxanthine phosphoribosyl-transferase-1 (Rn01527840_m1), FoxO1 (Rn01494868_m1), cyclin D1 (Rn00432359_m1), or Sprouty-2 (Spry2) (Rn02534289_s1). Real-time PCR analysis was conducted using the ABI PRISM 7700 Sequence Detector System (Applied Biosystems Canada). The comparative $C_{T}$ method was used to determine relative quantification of mRNA expression, using hypoxanthine phosphoribosyl-transferase and/or glyceraldehyde-3-phosphate dehydrogenase to normalize the amount of RNA per sample.

\section{In Vitro Experiments}

Microvascular endothelial cells isolated from rat EDL and TA muscles were cultured at $37^{\circ} \mathrm{C}$ and $6 \% \mathrm{CO}_{2}$ on $1.5 \%$ gelatin-coated flasks with Dulbecco's modified Eagle's medium supplemented with $10 \%$ fetal bovine serum, 1 $\mathrm{mmol} / \mathrm{L}$ sodium pyruvate, $200 \mathrm{mmol} / \mathrm{L}$ L-glutamine, 50 units of penicillin, and $0.5 \mathrm{mg} / \mathrm{ml}$ streptomycin as described previously. ${ }^{28}$ Cells were used for experiments between passages 4-7 and plated on type I collagen
(12.5 $\mu \mathrm{g}$ collagen $/ \mathrm{ml}$ coating buffer)-coated culture dishes at a density of $1 \times 10^{6}$ cells per $35-\mathrm{mm}$ dish overnight before stimulation with the following protocols. Cells were exposed to hypoxia (5\% oxygen) by incubation for 20 hours in an oxygen-regulated incubator. Cells were exposed to oxidative stress by culturing in the presence of hydrogen peroxide $(200,400,500$, or 800 $\mu \mathrm{mol} / \mathrm{L})$ for 1 hour. Generation of free radicals was confirmed using the radical-sensitive fluorescent dye 5-(AND-6)-CARBOXY-2', 7' -D 5-(and-6)-carboxy-2', 7'-difluorodihydrofluorescein diacetate (carboxy-H2DFFDA) (Invitrogen, Inc., Carlsbad, CA). Cells were loaded with the dye ( $5 \mu \mathrm{mol} / \mathrm{L}$ for 30 minutes) and treated with hydrogen peroxide $\left(\mathrm{H}_{2} \mathrm{O}_{2}\right)$ for 30 minutes, and then fluorescence was measured with a Wallac Victor microplate reader equipped with $480-\mathrm{nm}$ excitation and 520-nm emission filters. Cells were exposed to nutrient stress by incubation in serum-free, low glucose media (Optimem; Invitrogen, Inc.) for 24 to 48 hours. Shear stimulation was performed using the Bioptechs flow chamber 20 hours following plating of cells $(250,000)$ on collagen-coated coverslips. ${ }^{29}$ Cells were exposed to laminar flow $(\sim 15$ dynes $/ \mathrm{cm}^{2}$ ) for 2 hours or were maintained for the same duration under no-flow conditions. Following exposure to the stimulus for the specified time, cells were lysed and analyzed by Western blot. For all experiments using cultured endothelial cells, experiments were conducted a minimum of three times, on separate days, using independently prepared plates of cells.

\section{Immunoprecipitation}

Cell lysates or muscle extracts were incubated overnight with anti-Mdm2 (clone 2A10) antibody and then with protein A/G plus-agarose beads (Santa Cruz Biotechnology) for 2 to 3 hours at $4^{\circ} \mathrm{C}$. Supernatants were subjected to Western blot analysis as previously described to detect FoxO1 (Cell Signaling \#9454) or Mdm2 (2A10).

\section{Statistical Analysis}

Results were expressed as mean \pm SEM and analyzed by one-way analysis of variance and Tukey post-tests (Prism4; Graphpad Software Inc., La Jolla, CA). P values of less than 0.05 were considered statistically significant.

\section{Results}

\section{Ischemia-Induced HIF1 $\alpha$ and VEGF-A Expression Is Not Associated with an Increase in Capillary to Fiber Ratio in Rat Hind Limb Muscle}

Rats underwent ligation of the right common iliac artery or a corresponding sham operation and muscles were examined after 4, 7, or 14 days. We observed significant and sustained increases in hypoxia-sensitive $\mathrm{HIF} 1 \alpha$ and VEGF-A protein levels between 4 to 14 days after ligation (Figure 1, A and B), similar to previous reports. ${ }^{27,30}$ Despite the elevation in these pro-angiogenic factors, capillary to 
A
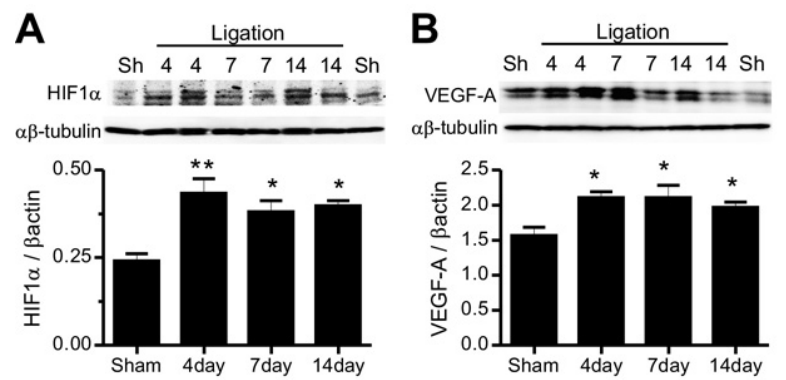

D
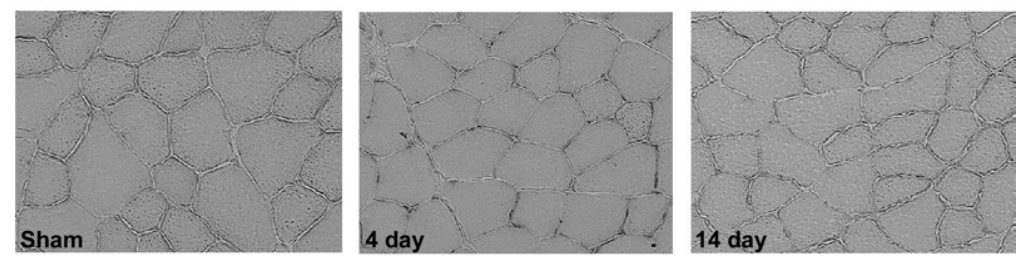

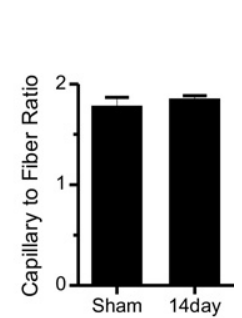

C

Figure 1. Ischemia induces HIF $1 \alpha$ and VEGF-A, but there is no increase in capillary number. Rats were subjected to hind limb ischemia by surgical ligation of the right common iliac artery or a corresponding sham (Sh) operation. Four, 7, or 14 days after ligation, rats were euthanized and muscles were removed for analysis. Protein was extracted from TA and EDL muscle was snap frozen for histology. A: HIF $1 \alpha$ protein levels were analyzed by Western blot. $\alpha \beta$-tubulin was probed as a loading control $\left(n=4 ;{ }^{*} P<0.05\right.$ versus 4 -day sham; ${ }^{* * *} P<0.01$ versus 4 -day sham). B: VEGF-A protein levels were analyzed by Western blot. $\alpha \beta$-tubulin was probed as a loading control $\left(n=4 ;{ }^{*} P<0.05\right.$ versus 4 -day sham). C: Capillary number was quantified in 14-day ischemic or sham-operated muscles and expressed relative to the muscle fiber number per field of view (capillary to fiber ratio) ( $n=4 ; P>0.05$ versus sham). D: Muscle histology was examined using toluidine blue-stained muscle crosssections. Muscle fiber morphology appeared well preserved, and no overt indications of inflammation or necrosis were observed. fiber ratio was not increased over the 14-day period (Figure $1 \mathrm{C}$ ), consistent with previous studies. ${ }^{27,30}$ Histologic examination of muscle morphology did not reveal overt signs of inflammatory cell infiltration, edema, or necrosis 4 or 14 days after ligation (Figure 1D).

\section{Ischemia Induces FoxO1 Expression in Hind Limb Muscle}

We next examined FoxO1 expression in ischemic hind limb muscles. Phospho-Ser256-FoxO1 and total FoxO1 protein levels were determined by Western blotting (Figure 2A). In ischemic muscles, FoxO1 protein levels were elevated significantly at the 4-day and 7-day time points (2.3-fold and twofold increase, respectively; $P<0.05$ versus controls that underwent a sham operation). Although the levels of phospho-Ser256-FoxO1 did not change at any experimental time point in comparison with sham values, the relative levels of phospho-FoxO1 compared with total FoxO1 protein levels were reduced substantively at 4 and 7 days after ligation (both $P<0.05$ versus sham). No differences in protein or mRNA levels of the examined genes were observed between the 4-day and 14-day control animals; therefore data from these muscle samples were pooled together to form a single sham group. FoxO1 protein was difficult to detect by immunofluorescent staining in EDL cross-sections from control animals (Figure 2B). In contrast, FoxO1 protein was strongly visible 7 days after artery ligation and colocalized with capillary staining (Figure 2C). Real-time PCR analysis revealed a transient small induction of FoxO1 mRNA in ischemic muscles with maximal expression at day 4 of ligation $(0.78 \pm 0.08$ in sham group versus $1.07 \pm 0.07$ after 4 days ligation; $P<0.05$ )

\section{Ischemia Alters Downstream Targets of FoxO1 Involved in Cell Cycle Regulation}

We examined the expression of downstream targets of FoxO1 involved in cell-cycle progression. The cell cycle inhibitor p27 ${ }^{\text {Kip1 }}$ was augmented in ischemic muscle extracts at day 4 and 7 after ligation ( $\sim$ twofold and 0.5-fold increase, respectively, $P<0.05$ versus sham), returning to sham values after 14 days (Figure 3A). Conversely, levels of cyclin D1 mRNA were attenuated significantly at 7 days after ligation compared with controls that underwent a sham operation ( $\sim 40 \%$ reduction, $P<$ 0.05 versus sham; Figure 3B). Levels of cleaved caspase-3 (an indicator of apoptosis) were not altered in the ischemic muscle (Figure 3C). Immunostaining for cleaved caspase-3 also did not reveal significant differences between ischemic and sham conditions (data not shown). We did not observe significant changes in eNOS protein level during the 2-week course of ischemia (Figure 3D). Spry2 mRNA, a downstream target of FoxO1 reported to influence angiogenesis, ${ }^{31}$ significantly decreased 4 days after the induction of ischemia (30\% reduction, $P<0.05$ versus sham; Figure 3E).

\section{Ischemia Alters the Phosphorylation of ERK and Akt, Two Pathways Involved in the Regulation of Fox01 Protein Level}

To further investigate the mechanisms responsible for FoxO1 up-regulation, we analyzed ERK, Akt, and p38 pathways, because each of them has been shown to regulate the stability or the activity of the transcription factor. ${ }^{22,23,32}$ Phosphorylation of mitogen-activated protein kinase ERK 1/2 was enhanced transiently (threefold) at 4 days after iliac artery ligation $(P<0.05$ versus sham; Figure 4A). In contrast, no significant change in p38 phosphorylation was observed (Figure 4B). Significant decreases in phospho-Akt levels (in relation to total Akt) were detected at day 4 and day 7 (60\% reduction for both time points; $P<0.05$ versus sham; Figure 4C). Phospho-Akt levels returned to levels seen in nonischemic muscle by 14 days after ligation. 
A
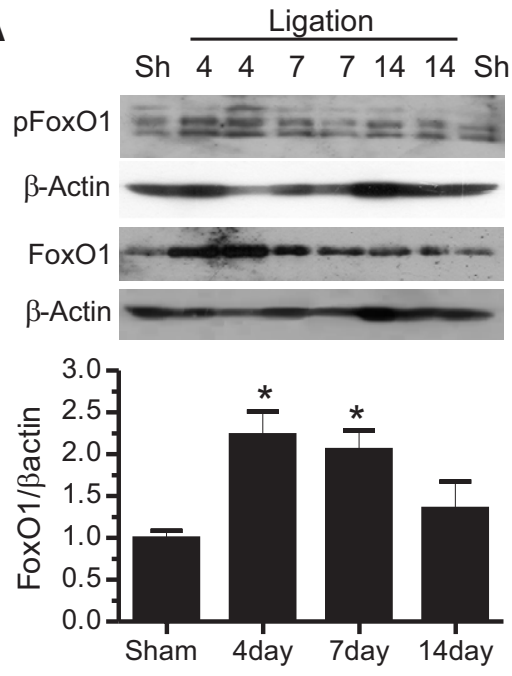
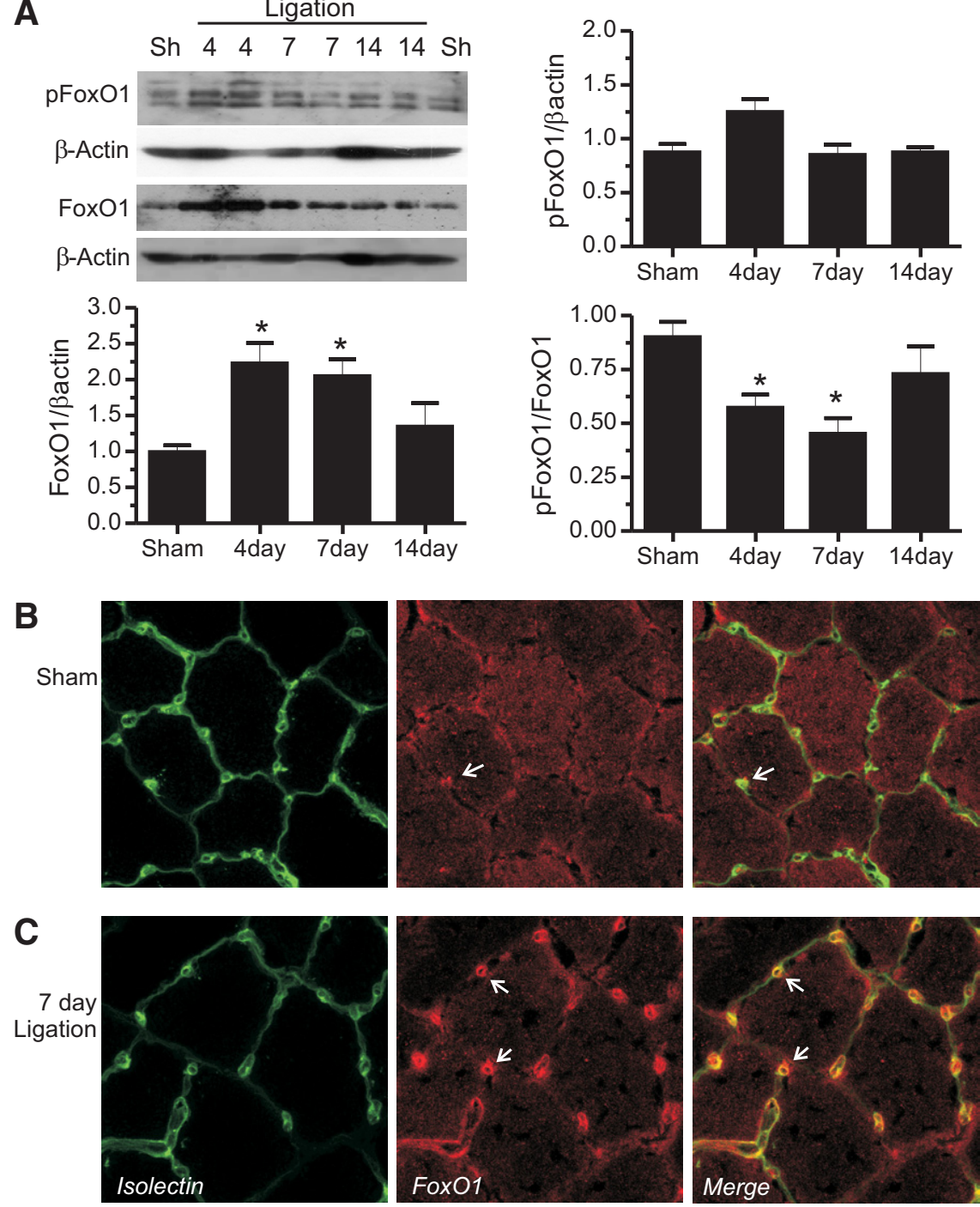

Figure 2. Evidence of increased levels of FoxO1 in ischemic muscle. Protein was extracted from rat TA muscle at 4,7 , or 14 days following induction of hind limb ischemia or a corresponding sham (Sh) operation. A: Phospho-(Ser256)-FoxO1 and total FoxO1 protein levels were analyzed by Western blot. Blots were stripped and reprobed for $\beta$-actin as a loading control. Phospho-FoxO1 and total FoxO1 protein levels were normalized to $\beta$-actin and expressed as a value relative to sham. Phospho-FoxO1 also was expressed relative to total FoxO1 $\left(n=4 ;{ }^{*} P<0.05\right.$ versus 4 -day sham). B and $\mathbf{C}$ : Frozen cross-sections of EDL from sham (B) or 7-day postligation (C) conditions were immunostained for FoxO1 (red) and counterstained with fluorescein isothiocyanate-conjugated Griffonia simplifica lectin (green) to label capillary endothelium. Muscle sections were viewed by confocal microscopy. Merged images are shown in the far right panels. Arrows point to FoxO1 staining co-localized with capillaries.

\section{Nutrient Deprivation, but not Hypoxic or Oxidative Stress, Enhances FoxO1 Expression in Cultured Endothelial Cells}

In the animal model of hind limb ischemia, restriction of blood flow generates numerous disturbances, including an inadequate oxygen supply, shortage of glucose and serum factors, and an accumulation of reactive oxygen species. $^{33,34}$ To further investigate the stimuli underlying FoxO1 up-regulation in response to ischemia, we evaluated FoxO1 expression in cultured microvascular endothelial cells that were exposed to ischemia-mimetic conditions. Low oxygen pressure $\left(5 \% \mathrm{O}_{2}\right.$ for 24 hours), which enhanced VEGF-A expression ( $\sim$ twofold increase versus control, $P<0.05$ ), did not affect FoxO1 protein expression in endothelial cells (Figure 5A). Treatment of cells with $\mathrm{H}_{2} \mathrm{O}_{2}$ induced a concentration-dependent increase in free radicals as estimated by the oxidation of the dichlorodihydrofluorescein into the fluorescent dichlorofluorescein $[1.2 \pm 0.01$-fold increase $(200 \mu \mathrm{mol} / \mathrm{L})$ to 3.0 \pm 0.09 -fold increase $(800 \mu \mathrm{mol} / \mathrm{L})$ vs untreated cells, $P<$
0.05] and stimulated intense phosphorylation of oxidative stress-sensitive kinase ERK $1 / 2$ but did not modulate FoxO1 protein levels (Figure 5B). Conversely, prolonged nutrient stress for 24 hours significantly elevated FoxO1 protein expression by twofold versus control $(P<0.05)$ in cultured endothelial cells (Figure 5C).

\section{In the Absence of Shear Stimulation, Cultured Endothelial Cells Are Characterized by Low Levels of Akt Phosphorylation and Elevated FoxO1 Protein}

Ischemia also induces a reduction in capillary blood flow and shear stress, which may affect endothelial cell gene expression. Thus we compared Akt phosphorylation and FoxO1 expression in endothelial cells maintained under no-flow or shear-stimulated conditions. In agreement with previous reports, ${ }^{35}$ we observed that cells kept under no-flow conditions had $65 \%$ less Akt phosphorylation on residue serine $473 \mathrm{com}$ - 
A

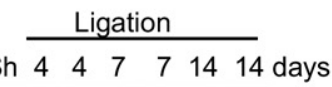

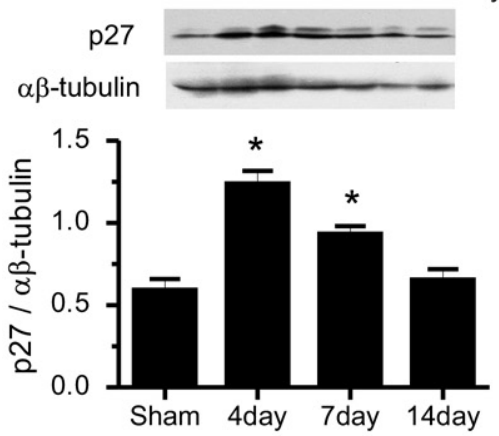

D
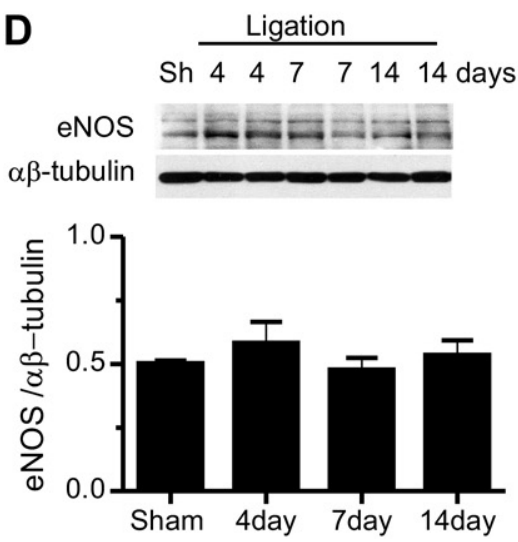

B

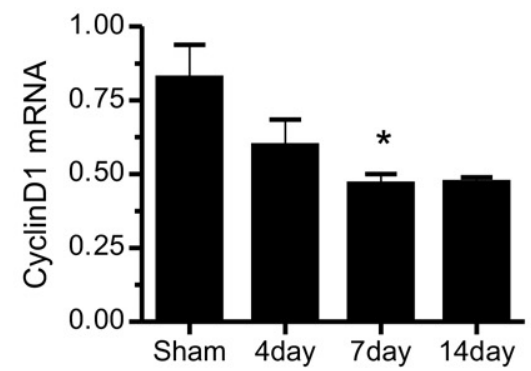

E

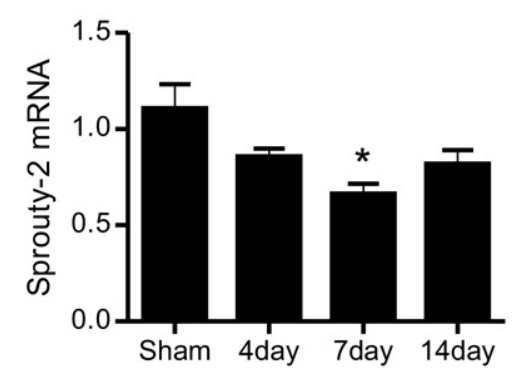

C
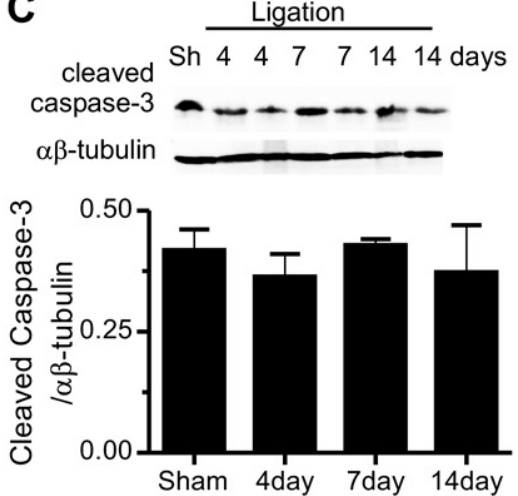

Figure 3. Responsiveness of putative FoxO target genes to ischemia. Protein or RNA was extracted from rat TA and EDL, respectively, at 4,7 , or 14 days following induction of hind limb ischemia or a corresponding sham (Sh) operation. A: p2 $7^{\text {Kip } 1}$ protein level was analyzed by Western blot. Blots were stripped and reprobed for $\alpha / \beta$-tubulin as a loading control. p27 levels were normalized to $\alpha / \beta$-tubulin and expressed relative to sham ( $n=4 ;{ }^{*} P<0.05$ versus sham). B: Cyclin D1 mRNA expression was analyzed by real-time PCR, with values normalized to housekeeping genes GAPDH and HPRT ( $n=4 ;{ }^{*} P<0.05$ versus sham) C: Levels of cleaved caspase-3 were analyzed by Western blot. Blots were stripped and reprobed for $\alpha / \beta$-tubulin as a loading control $(n=4 ; P>0.05)$. D: eNOS protein expression was analyzed by Western blot and normalized to $\alpha / \beta$ tubulin as a loading control $(n=4 ; P>0.05)$. E: Spry-2 mRNA expression was analyzed by real-time PCR, with values normalized to housekeeping genes glyceraldehyde3-phosphate dehydrogenase and hypoxanthine phosphoribosyl-transferase $\left(n=4 ;{ }^{*} P<0.05\right.$ versus sham). pared with cells subjected to 2 hours of shear stimulation $(P<$ 0.05 versus shear-stimulated cells; Figure 6A). Conversely, FoxO1 protein expression was increased significantly in microvascular endothelial cells maintained under no-flow conditions (1.8-fold increase, $P<0.05$ versus shear-stimulated cells; Figure 6B). FoxO1 mRNA expression was not different between shear-stimulated or no-flow endothelial cells (1.0 versus $0.81 \pm 0.14$ (30 minutes shear) or 1.0 versus $3.0 \pm 0.87$ ( 2 hours shear); $n=4, P>0.05$ shearstimulated versus time-matched no-flow cells). Akt activation is associated with enhanced proteasomal degradation via ubiquitin ligase pathways. ${ }^{24}$ The E3 ubiquitin ligase Mdm2 is a substrate for Akt and was reported recently to mediate FoxO ubiquitination. ${ }^{22,23}$ We detected phosphoSer166 (active) Mdm2 in shear-stimulated endothelial cells; however, this phosphorylation substantially decreased in
A

$\begin{array}{llllllll}\text { Sh } & 4 & 4 & 7 & 7 & 14 & 14 \text { days }\end{array}$
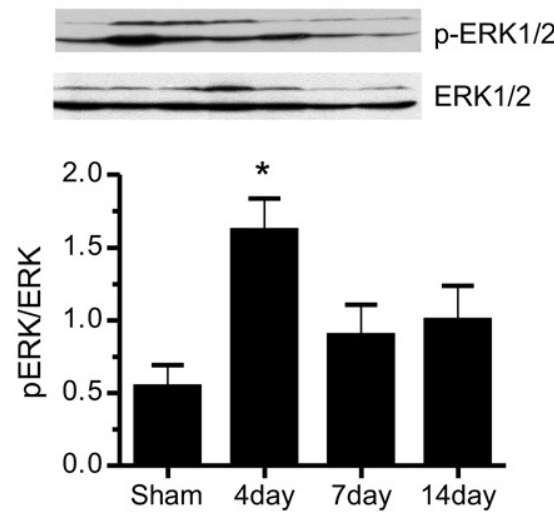

B Sh $\begin{array}{llllll}4 & 4 & 7 & 7 & 14 & 14 \\ \text { days }\end{array}$
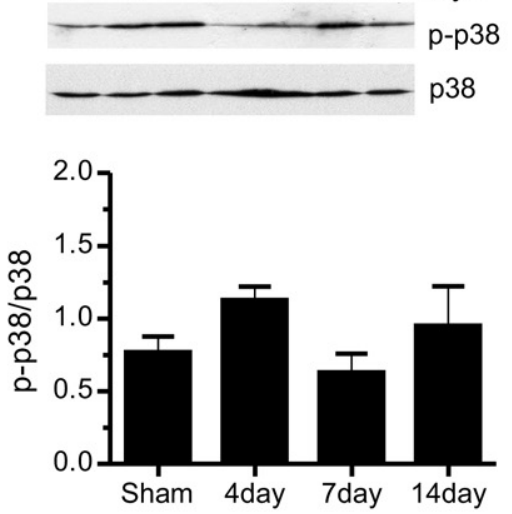

C Sh $4 \begin{array}{llll} & 7 & 14 & \text { Sh days }\end{array}$
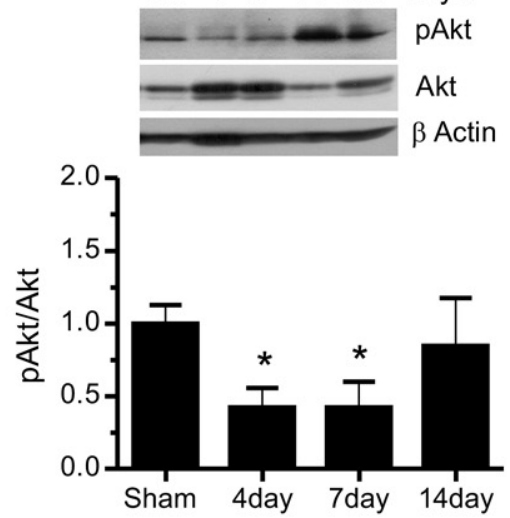

Figure 4. ERK 1/2, p38 MAPK, and Akt phosphorylation in response to ischemia. Protein was extracted from rat tibialis anterior at 4, 7 , or 14 days following induction of hind limb ischemia or a corresponding sham (Sh) operation. A: Phospho-ERK $1 / 2$ and total ERK $1 / 2$ protein levels were analyzed by Western blot. Phospho-ERK $1 / 2$ was expressed relative to total ERK $1 / 2\left(n=4 ;{ }^{*} P<0.05\right.$ versus sham). B: Phospho-p38 and total p38 protein levels were analyzed by Western blot. Phospho-p38 was expressed relative to total p38 ( $n=4$; not significant). C: Phospho-(Ser473)-Akt levels were normalized to total Akt in each sample and expressed as a value relative to the 4 -day $\operatorname{sham}\left(n=3 ;{ }^{*} P<0.05\right.$ versus 4 -day sham).). $\beta$-actin levels are shown to indicate loading. 

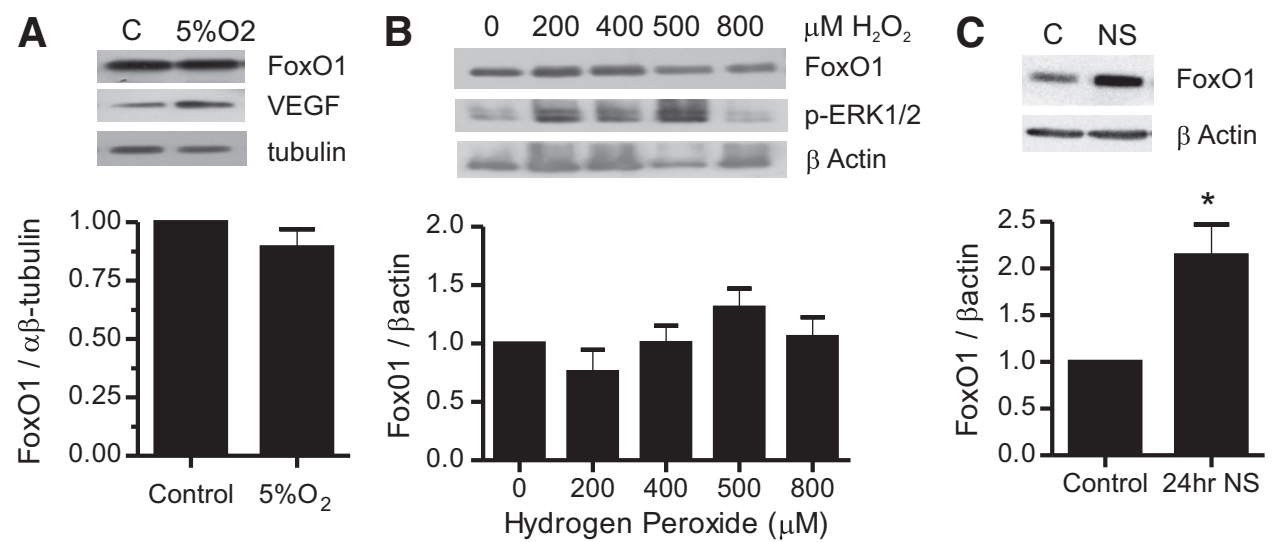

Figure 5. FoxO1 protein levels in cultured endothelial cells are regulated by nutrient stress but not by hypoxia or oxidative stress. A: Rat microvascular endothelial cells were cultured in hypoxic conditions ( $5 \%$ oxygen) for 24 hours. FoxO1 protein was quantified by Western blotting of whole cell lysate and normalized to $\alpha / \beta$-tubulin as a loading control. VEGF-A protein was assessed as a positive control for the effects of hypoxia ( $n=3 ; P>0.05$ versus normoxic control). B: Microvascular endothelial cells were subjected to increased oxidative stress by culturing in the presence of $\mathrm{H}_{2} \mathrm{O}_{2}$ for 1 hour. FoxO1 was analyzed by Western blot and normalized to $\beta$-actin $(n=3)$. Phospho-ERK $1 / 2$ was analyzed to demonstrate the efficacy of $\mathrm{H}_{2} \mathrm{O}_{2}$ treatment. C: Microvascular endothelial cells were subjected to increased nutrient stress (NS) by culturing in Optimem (reduced serum/low glucose media) for 24 hours. FoxO1 was analyzed by Western blot and normalized to $\beta$-actin $\left(n=3 ;{ }^{*} P<0.0 .5\right.$ versus control $)$

cells maintained under no-flow conditions (25-fold decrease, $P<0.05$ versus shear-stimulated cells; Figure 6C).

\section{FoxO1 Interaction with Mdm2 Is Reduced in Ischemic Muscle}

Based on our observation of reduced Akt phosphorylation under ischemic conditions, we postulated that Mdm2 phosphorylation also would be reduced under these conditions. We observed that phospho-(Ser166)-Mdm2 was reduced substantially in response to nutrient stress ( $~ 50 \%$ reduction, $P<0.05$ versus time-matched control; Figure 7A). Conversely, serum stimulation of starved cells resulted in increased phospho-(Ser166)-Mdm2 levels and reduced FoxO1 protein expression, correlating also with the appearance of a higher molecular weight band (marked by asterisk on Figure 7B). Treatment of serumstimulated cells with the proteasomal inhibitor MG132 augmented FoxO1 protein level, particularly that of the higher molecular weight band (Figure 7C). Given the inverse relationship observed between FoxO1 protein level and Mdm2 phosphorylation under various experimental conditions, we looked for direct evidence of Mdm2-FoxO1 interaction. FoxO1 protein was detectable following immunoprecipitation of Mdm2 from serumstarved cell lysates, and serum stimulation of these cells enhanced this interaction (Figure 7D). We also found that the amount of phospho-(Ser166)-Mdm2 was reduced significantly at day 4 of ischemia (65\% reduction, $P<0.05$ versus controls that underwent a sham operation; Figure $7 E)$. This decline also was noticeable but not statistically significant at day 7 (48\% reduction; $P=0.065$ ). Consistent with our observations in cultured endothelial cells that the extent of Mdm2-FoxO1 interaction correlates directly with Mdm2 phosphorylation, immunoprecipitation of Mdm2 from ischemic muscles pulled down a reduced amount of FoxO1 compared with sham muscles (Figure 7F).

\section{Discussion}

Our study provides novel evidence of the enhanced endogenous expression of the transcription factor FoxO1 within the microcirculation of hind limb skeletal muscles in response to an ischemic insult. The increased level of FoxO1 correlates with the modulation of cell cycle regulators and thus may contribute to the inhibition of recovering angiogenesis. Using cultured endothelial cells, we provide evidence that nutrient deprivation or lack of shear stress is
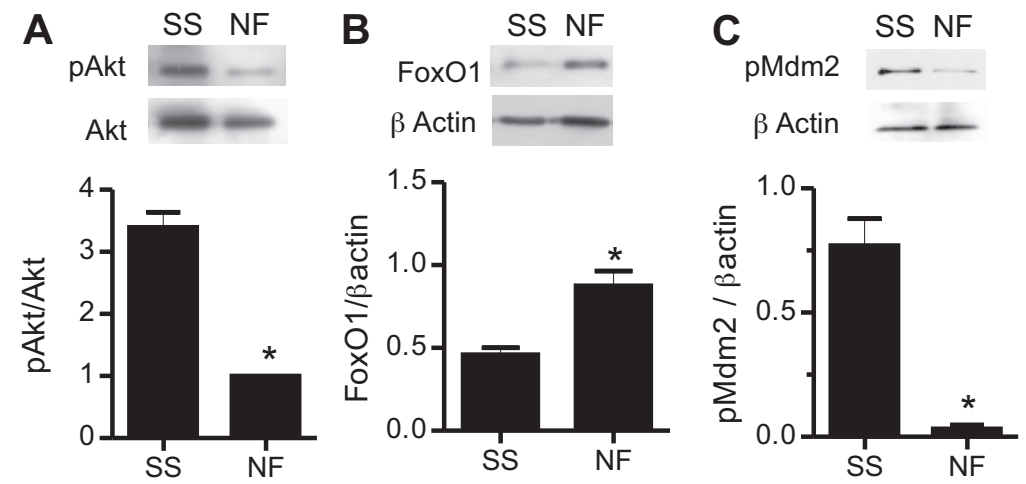

Figure 6. Reduced Akt activation under no-flow conditions correlates with increased FoxO1 protein and reduced phosphorylation of Mdm2. Rat microvascular endothelial cells were maintained under no flow (NF) or exposed to continuous shear stress $\left(15\right.$ dynes $/ \mathrm{cm}^{2}$ ) for 1 hour then lysed for analysis by Western blot. A: Phospho-(Ser473) Akt was normalized to total Akt $\left(n=3 ;{ }^{*} P<0.05\right.$ versus shear-stimulated cells). B: FoxO1 was normalized to $\beta$-actin $\left(n=3 ;{ }^{*} P<0.05\right.$ versus shear-stimulated cells). C: Phospho-(Ser166)-Mdm2 was normalized to $\beta$-actin $\left(n=3 ;{ }^{*} P<0.05\right.$ versus shearstimulated cells) 
A

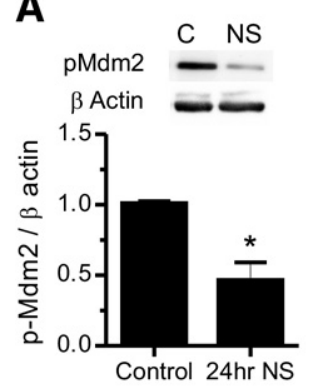

B

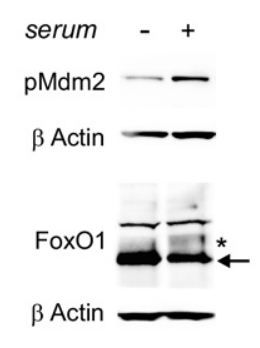

C

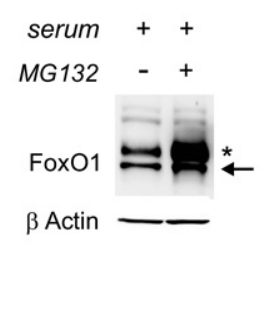

D

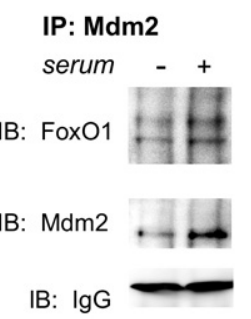

E

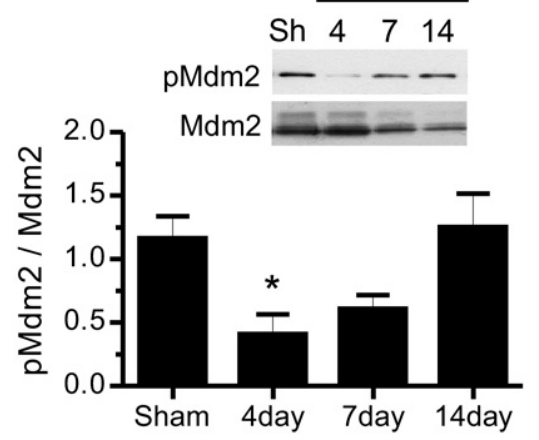

$\mathbf{F}$

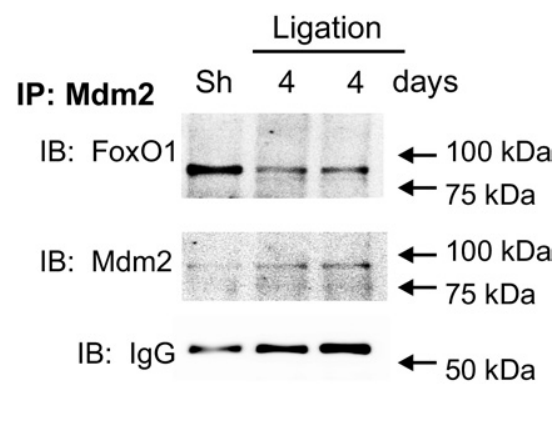

Figure 7. Mdm2-FoxO1 interaction correlates with Mdm2 phosphorylation. Nutrient stressed cells or ischemic muscle have reduced Mdm2 phosphorylation and Mdm2-FoxO1 interaction. A: Microvascular endothelial cells were subjected to increased nutrient stress (NS) by culturing in Optimem for 24 hours Phospho-(Ser166)-Mdm2 was analyzed by Western blot and normalized to $\beta$-actin $\left({ }^{*} P<0.05, n=4\right)$. $\mathbf{B}$. Microvascular endothelial cells were subjected to low serum ( $2 \%$ fetal bovine serum) media for 24 hours followed by serum stimulation ( $10 \%$ fetal bovine serum) for 90 minutes. Phospho-(Ser160)-Mdm2 and FoxO1 protein levels were detected by Western blot. The asterisk points to a higher molecular weight band appearing over the FoxO1 protein band (arrow). $\beta$-actin was probed as a loading control. (C) MG132 $(10 \mu \mathrm{mol} / \mathrm{L})$ or vehicle was added at the time of serum stimulation. FoxO1 protein was detected by Western blot. The asterisk points to a higher molecular weight band appearing over the FoxO1 protein band (arrow). $\beta$-actin was probed as a loading control. D: $\mathrm{Mdm} 2$ was immunoprecipitated from serum-starved cells or those stimulated with serum (90 minutes) after starvation. FoxO1 was immunoblotted, followed by reprobing for $\mathrm{Mdm} 2$ and IgG as a loading control. E: Proteins were extracted from tibialis anterior of rats at 4,7 , or 14 days following induction of hind limb ischemia or a corresponding sham (Sh) operation. Phospho-(Ser166)-Mdm2 expression was analyzed by Western blot and normalized to $\mathrm{Mdm} 2\left({ }^{*} P<0.05\right.$ versus sham; $\left.n=4\right)$. F: $\mathrm{Mdm} 2$ was immunoprecipitated from lysates of sham or 4-day ligated TA muscle. Blots were probed for FoxO1 and then for Mdm2 and IgG as loading controls. Blots in $\mathrm{B}-\mathrm{D}$ and $\mathrm{F}$ are representative of multiple experiments ( $n=3$ to 4 ) a sufficient stimulus to promote enhanced levels of FoxO1. Furthermore, we provide novel evidence of endogenous Mdm2-FoxO1 interaction within cultured endothelial cells as well as in vivo in muscle tissue. Our data suggest that reduced phosphorylation of Akt/Mdm2 may contribute to the increased levels of FoxO1 in response to ischemia.

\section{FoxO1 Is a Factor Associated with Ischemia- Mediated Alterations in Muscle Microcirculation}

While a potential role for the FoxO family during ischemia has been proposed previously, the mechanisms by which these proteins regulate the process of neovascularization in ischemic tissue remains largely unknown. Potente et al assessed postischemia revascularization in skeletal muscles of transgenic FoxO3a ${ }^{-1-}$ mice. The authors observed a significant enhancement of postischemia limb perfusion relative to wild-type animals, and this enhancement was associated with enhanced capillary density. ${ }^{16}$ However, they did not examine endogenous levels of FoxO proteins in the ischemic tissue. Paik et al demonstrated that conditional deletion of FoxO1 was sufficient to drive development of hemangiomas due to progressive over-proliferation of endothelial cells. ${ }^{31}$ It also was shown that FoxO1 over-expression in endothelial cells triggers cellcycle arrest at G1 and inhibition of DNA synthesis. ${ }^{18}$

Here, we demonstrated a significant increase in FoxO1 protein level co-localized to microvessels within ischemic muscles, suggesting that the increased amount of FoxO1 protein may contribute to the repression of angiogenesis within the ischemic environment. Considering that an effective angiogenic cascade requires endothelial cells to leave their normal quiescent state and enter the cell cycle, elevated levels of cyclin-dependent kinase inhibitors can critically influence the process of capillary growth. For example, over-expression of $\mathrm{p} 27^{\mathrm{Kip} 1}$ seems sufficient to reduce capillary density and to impair blood flow recovery in a murine model of hind limb ischemia. ${ }^{36}$ Our results showed an increased expression of $\mathrm{p} 27^{\mathrm{Kip} 1}$ (which is an established transcriptional target of FoxO1) at days 4 and 7 after ligation, correlating closely with the time course of increase in FoxO1 protein. Our findings are congruent with a previous report showing induction of FoxO transcriptional activation in mouse hearts subjected to ischemia-reperfusion. ${ }^{37}$ The same study reported that transgenic mice over-expressing cardiac FoxO1 were characterized by decreased cardiomyocyte cell proliferation through up-regulation of $\mathrm{p} 21^{\mathrm{Cip} 1}$ and $\mathrm{p} 27^{\mathrm{Kip} 1}$ genes. ${ }^{37} \mathrm{We}$ also observed a decreased level of cyclin D1 mRNA in ischemic muscles, consistent with previous reports of FoxO-dependent repression of cyclin D1 transcription. ${ }^{38-40}$ Conversely, levels of cleaved caspase-3 did not change, indicating no elevation in apoptotic signaling. We did not detect an increase in Spry2 mRNA (a negative regulator of the MEK pathway that may be controlled by FoxO transcription factors ${ }^{31}$ ) in ischemic muscles. Although unexpected, this finding might be explained by the fact that Spry2 gene expression also is regulated by Ets-1. ${ }^{41}$ We measured a transient decrease in Ets-1 mRNA in response to ischemia (data not shown), and Spry2 mRNA appears to follow the same expression pattern. We did not observe any change in eNOS levels in ischemic muscle in contrast to in vitro studies showing in cultured cells that over-expression of either FoxO1 or FoxO3a repressed eNOS expression. ${ }^{16}$ However, our results are consistent with a previous study using the rat iliac artery ligation model of ischemia that reported no change in eNOS protein level despite elevated levels of VEGF-A. ${ }^{42}$ 


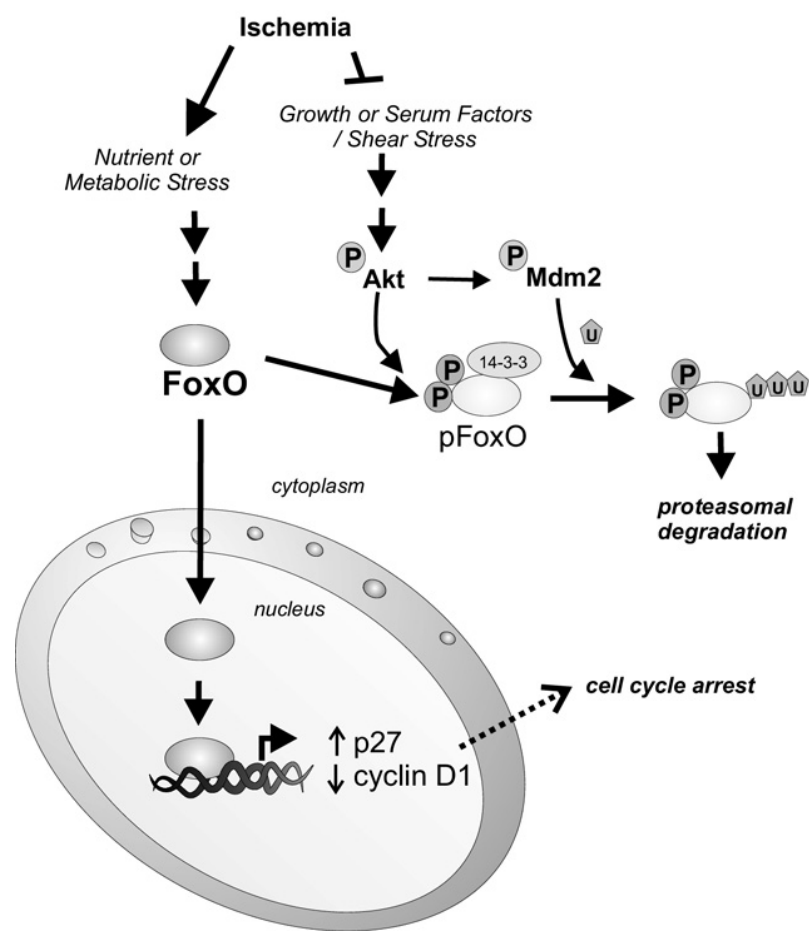

Figure 8. Putative regulation of the FoxO1 pathway in response to ischemia. Ischemia induces metabolic stress and reduces signaling through growth factors and shear stress receptors. Decreased activation of Akt results in lower phosphorylation of FoxO1 and also lower phosphorylation of the E3 ubiquitin ligase $\mathrm{Mdm} 2$, together resulting in reduced degradation of FoxO1 protein. Nutrient stress also may evoke signals that induce FoxO1 nuclear localization, where it can act as a transcriptional regulator. Cell cycle arrest through decreased expression of activators (cyclin D1) and enhanced production of inhibitors ( $\left.\mathrm{p} 27^{\mathrm{Kip} 1}\right)$ is one outcome of FoxO1 activation that may impede a recovering angiogenic response in muscle tissue.

Together, these results point to the complexities associated with understanding FoxO1 function within a multicellular environment that is responding to a pleiotropic stimulus. The role of FoxO in regulating the expression of these and other genes in response to ischemia requires further elucidation, because the pattern of gene regulation likely is dependent on the extent of FoxO up-regulation and the levels of transcriptional co-regulators.

\section{Role of Nutrient Stress and Shear Stress in the Regulation of FoxO1}

We examined upstream regulation of FoxO1 to further our understanding of the mechanisms underlying the ischemia-dependent increase in FoxO1 protein. While numerous kinases are known to regulate FoxO1 protein stabilization and functional activity by phosphorylation, ${ }^{43}$ we found that the decline in phosphorylation of Akt, but not that of ERK $1 / 2$ or p38 MAPK, fit closely with the timecourse increase in FoxO1 protein. This finding is consistent with the established mechanism of Akt stimulating FoxO1 cytoplasmic sequestration and subsequent proteasomal degradation. ${ }^{10,19}$ Our findings in cultured endothelial cells further supported this relationship, because nutrient stress or lack of a shear stress stimulus promoted a reduced state of Akt phosphorylation and enhanced FoxO1 protein levels. Our data suggest that nutrient stress and reduction of a shear stress stimulus may both contribute to the elevation in FoxO1 protein observed in ischemic muscles.

Interestingly, the pattern of Mdm2 phosphorylation correlated positively with that of Akt phosphorylation, increasing in response to shear stimulation and decreasing during nutrient or ischemic stress. Brenkman and colleagues previously reported that Mdm2 induced polyubiquitination of FoxO4 and that Akt phosphorylation of FoxO was required for $\mathrm{Mdm} 2-\mathrm{FoxO}$ interaction and the subsequent ubiquitination/degradation of FoxO4. ${ }^{44}$ Consistent with their observations, we found that the extent of Mdm2-FoxO1 interaction correlated positively with Mdm2 phosphorylation both in cultured endothelial cells and in ischemic muscles. While Mdm2-FoxO1 interactions have been reported in a cell-free system, ${ }^{22}$ our data provide novel in vivo measures of FoxO1-Mdm2 interaction and suggest that Mdm2 may act as a critical regulator of FoxO1 protein level in endothelial cells.

\section{Conclusion}

This study has demonstrated for the first time that the development of ischemia in skeletal muscle is associated with up-regulation of FoxO1. Enhanced expression of FoxO1 during ischemia correlates with reduced Akt phosphorylation and may be triggered by nutrient stress and reduction of shear stress. Our study provides the first evidence of endogenous Mdm2-FoxO1 interactions within microvascular endothelial cells and in ischemic muscle. Because Mdm2 ubiquitin ligase activity itself is regulated via Akt-dependent phosphorylation, ${ }^{21}$ Akt appears to be a critical regulator of FoxO1 function at multiple levels. In the ischemic environment, FoxO1 escapes these repressive signals as illustrated by the decreased interaction between Mdm2 and FoxO1 observed in ischemic muscle postligation, which correlated with increased total levels of FoxO1 and enhanced expression of cell cycle inhibitor p27 ${ }^{\mathrm{Kip} 1}$ (Figure 8). In light of the established effects of FoxO1 on cell cycle regulation, it is compelling to consider this transcription factor as a modulator able to override the pro-angiogenic signaling pathways. Further clarification of FoxO protein regulation and function during ischemic episodes may lay the foundation for the successful application of clinical therapies for ischemia.

\section{References}

1. Ferrara N: Vascular endothelial growth factor: basic science and clinical progress. Endocrine Rev 2004, 25:581-611

2. Rajagopalan S, Olin J, Deitcher S, Pieczek A, Laird J, Grossman PM Goldman CK, McEllin K, Kelly R, Chronos N: Use of a constitutively active hypoxia-inducible factor-1alpha transgene as a therapeutic strategy in no-option critical limb ischemia patients: phase I doseescalation experience. Circulation 2007, 115:1234-1243

3. Yla-Herttuala S, Alitalo K: Gene transfer as a tool to induce therapeutic vascular growth. Nat Med 2003, 9:694-701

4. Henry TD, Annex BH, McKendall GR, Azrin MA, Lopez JJ, Giordano FJ, Shah PK, Willerson JT, Benza RL, Berman DS, Gibson CM, Bajamonde A, Rundle AC, Fine J, McCluskey ER, Boisjolie C, Landis D, 
Martz C, Donahue L, Barry MB, Costigan M, Van Dijk J, Gheorghiu M, Weiglet L, Guzman I, Hendel R, Toth B, Ritondo C, Valente R, Pierce B, Smith J, Miele R, Rocha-Singh K, Laswell KS, Giacomini J, Sharma V. Ellis S, Comella K, From A, Bass L, Cooke J, Rockson S, Lissin L, Willerson J, Harlan M, Kleiman N, Maresh K, Marshall J, Moehring K, Kraft P, Fox M, Faxon D, Gutierrez I, Sarembock I, Snyder L, Steingart R, Parker S, Strumpf R, Spooner S, Marshall J, Lane A, Cloutier J, Weisshaar D, Stewart D, Hinohara T, Johnson M, Luce C, Tu T, Bisio D, Kuczma J, Klein R, Neider M, Meuer S, Ryan J, Lewin H, Miranda L. The VIVA trial: vascular endothelial growth factor in ischemia for vascular angiogenesis. Circulation 2003, 107:1359-1365

5. Gupta R, Tongers J, Losordo DW: Human studies of angiogenic gene therapy. Circ Res 2009, 105:724-736

6. Sun L, Bai Y, Du G: Endothelial dysfunction-an obstacle of therapeutic angiogenesis. Ageing Res Rev 2009, 8:306-313

7. Burgering BMT: A brief introduction to FOXOlogy. Oncogene 2008 , 27:2258-2262

8. Salih DA, Brunet A: FoxO transcription factors in the maintenance of cellular homeostasis during aging. Curr Opin Cell Biol 2008, 20:126-136

9. Dansen TB, Burgering BM: Unravelling the tumor-suppressive functions of FOXO proteins. Trends Cell Biol 2008, 18:421-429

10. Papanicolaou KN, Izumiya $Y$, Walsh K: Forkhead transcription factors and cardiovascular biology. Circ Res 2008, 102:16-31

11. Burgering BM, Medema RH: Decisions on life and death: fOXO Forkhead transcription factors are in command when PKB/Akt is off duty. J Leukoc Biol 2003, 73:689-701

12. Tran $\mathrm{H}$, Brunet A, Griffith EC, Greenberg ME: The many forks in FOXO's road. Sci STKE 2003, 172:RE5

13. Hosaka T, Biggs WH, III, Tieu D, Boyer AD, Varki NM, Cavenee WK Arden KC: Disruption of forkhead transcription factor (FOXO) family members in mice reveals their functional diversification. Proc Natl Acad Sci USA 2004, 101:2975-2980

14. Furuyama T, Kitayama K, Shimoda Y, Ogawa M, Sone K, YoshidaAraki K, Hisatsune H, Nishikawa S, Nakayama K, Nakayama K, Ikeda $\mathrm{K}$, Motoyama N, Mori N: Abnormal angiogenesis in Foxo1 (Fkhr)deficient mice. J Biol Chem 2004, 279:34741-34749

15. Daly C, Wong V, Burova E, Wei Y, Zabski S, Griffiths J, Lai KM, Lin HC, loffe E, Yancopoulos GD, Rudge JS: Angiopoietin-1 modulates endothelial cell function and gene expression via the transcription factor FKHR (FOXO1). Genes Dev 2004, 18:1060-1071

16. Potente M, Urbich C, Sasaki K, Hofmann WK, Heeschen C, Aicher A, Kollipara R, DePinho RA, Zeiher AM, Dimmeler S: Involvement of Foxo transcription factors in angiogenesis and postnatal neovascularization. J Clin Invest 2005, 115:2382-2392

17. Urbich C, Knau A, Fichtlscherer S, Walter DH, Bruhl T, Potente M, Hofmann WK, de Vos S, Zeiher AM, Dimmeler S: FOXO-dependent expression of the proapoptotic protein Bim: pivotal role for apoptosis signaling in endothelial progenitor cells. FASEB J 2005, 19:974-976

18. Abid M, Guo S, Minami T, Spokes KC, Ueki K, Skurk C, Walsh K, Aird WC: Vascular endothelial growth factor activates PI3K/Akt/forkhead signaling in endothelial cells. Arterioscler Thromb Vasc Biol 2004, 24:294-300

19. Aoki M, Jiang $\mathrm{H}$, Vogt PK: Proteasomal degradation of the FoxO1 transcriptional regulator in cells transformed by the P3k and Akt oncoproteins. Proc Natl Acad Sci USA 2004, 101:13613-13617

20. Huang H, Regan KM, Wang F, Wang D, Smith DI, van Deursen JMA, Tindall DJ: Skp2 inhibits FOXO1 in tumor suppression through ubiquitinmediated degradation. Proc Natl Acad Sci USA 2005, 102:1649-1654

21. Zhou BP, Liao Y, Xia W, Zou Y, Spohn B, Hung MC: HER-2/neu induces p53 ubiquitination via Akt-mediated MDM2 phosphorylation. Nat Cell Biol 2001, 3:973-982

22. Fu W, Ma Q, Chen L, Li P, Zhang M, Ramamoorthy S, Nawaz Z, Shimojima $T$, Wang $H$, Yang $Y$, Shen Z, Zhang $Y$, Zhang $X$, Nicosia SV, Zhang Y, Pledger JW, Chen J, Bai W: MDM2 acts downstream of p53 as an E3 ligase to promote FOXO ubiquitination and degradation. J Biol Chem 2009, 284:13987-14000

23. Yang JY, Zong CS, Xia W, Yamaguchi $\mathrm{H}$, Ding Q, Xie X, Lang JY, Lai CC, Chang CJ, Huang WC, Huang H, Kuo HP, Lee DF, Li LY, Lien HC, Cheng X, Chang KJ, Hsiao CD, Tsai FJ, Tsai CH, Sahin AA, Muller WJ, Mills GB, Yu D, Hortobagyi GN, Hung MC: ERK promotes tumorigenesis by inhibiting FOXO3a via MDM2-mediated degradation. Nat Cell Biol 2008, 10:138-148

24. Huang $\mathrm{H}$, Tindall DJ: Dynamic FoxO transcription factors. J Cell Sci 2007, 120:2479-2487
25. Chlench S, Mecha Disassa N, Hohberg M, Hoffmann C, Pohlkamp T, Beyer G, Bongrazio M, Silva-Azevedo L, Baum O, Pries AR, Zakrzewicz A: Regulation of Foxo-1 and the angiopoietin-2/Tie2 system by shear stress. FEBS Lett 2007, 581:673-680

26. Goettsch W, Gryczka C, Korff T, Ernst E, Goettsch C, Seebach J, Schnittler HJ, Augustin HG, Morawietz H: Flow-dependent regulation of angiopoietin-2. J Cell Physiol 2008, 214:491-503

27. Milkiewicz M, Hudlicka O, Verhaeg J, Egginton S, Brown MD: Differential expression of Flk-1 and Flt-1 in rat skeletal muscle in response to chronic ischaemia: favourable effect of muscle activity. Clin Sci (Lond) 2003, 105:473-482

28. Han XY, Boyd PJ, Colgan S, Madri JA, Haas TL: Transcriptional up-regulation of endothelial cell matrix metalloproteinase-2 in response to extracellular cues involves GATA-2. J Biol Chem 2003, 278:47785-47791

29. Milkiewicz M, Kelland C, Colgan S, Haas TL: Nitric oxide and p38 MAP Kinase mediate shear stress-dependent inhibition of MMP-2 production in microvascular endothelial cells. J Cell Physiol 2006, 208:229-237

30. Milkiewicz M, Hudlicka O, Shiner R, Egginton S, Brown MD: Vascular endothelial growth factor mRNA and protein do not change in parallel during non-inflammatory skeletal muscle ischaemia in rat. J Physiol 2006, 577:671-678

31. Paik JH, Kollipara R, Chu G, Ji H, Xiao Y, Ding Z, Miao L, Tothova Z, Horner JW, Carrasco DR, Jiang S, Gilliland DG, Chin L, Wong WH, Castrillon DH, DePinho RA: FoxOs are lineage-restricted redundant tumor suppressors and regulate endothelial cell homeostasis. Cell 2007, 128:309-323

32. Puthanveetil P, Wang Y, Wang F, Kim MS, Abrahani A, Rodrigues B: The increase in cardiac pyruvate dehydrogenase kinase-4 after shortterm dexamethasone is controlled by an Akt-p38-forkhead box other factor-1 signaling axis. Endocrinology 2010, 151:2306-2318

33. Pipinos II, Swanson SA, Zhu Z, Nella AA, Weiss DJ, Gutti TL, McComb RD, Baxter BT, Lynch TG, Casale GP: Chronically ischemic mouse skeletal muscle exhibits myopathy in association with mitochondrial dysfunction and oxidative damage. Am J Physiol Regul Integr Comp Physiol 2008, 295:R290-R296

34. Pipinos II, Judge AR, Selsby JT, Zhen Z, Swanson SA, Nella AA, Dodd SL: Basic science review: the myopathy of peripheral arterial occlusive disease: part 2. Oxidative Stress Neuropathy Shift Muscle Fiber Type Vasc Endovasc Surg 2008, 42:101-112

35. Dimmeler S, Fleming I, Fisslthaler B, Hermann C, Busse R, Zeiher AM: Activation of nitric oxide synthase in endothelial cells by Akt-dependent phosphorylation. Nature 1999, 399:601-605

36. Goukassian D, Diez-Juan A, Asahara T, Schratzberger P, Silver M, Murayama T, Isner JM, Andres V: Overexpression of p27(Kip1) by doxycyclineregulated adenoviral vectors inhibits endothelial cell proliferation and migration and impairs angiogenesis. FASEB J 2001, 15:1877-1885

37. Sengupta A, Molkentin JD, Yutzey KE: FoxO transcription factors promote autophagy in cardiomyocytes. J Biol Chem 2009, 284:28319-28331

38. Ramaswamy S, Nakamura N, Sansal I, Bergeron L, Sellers WR: A novel mechanism of gene regulation and tumor suppression by the transcription factor FKHR. Cancer Cell 2002, 2:81-91

39. Schmidt M, Fernandez de Mattos S, van der Horst A, Klompmaker R, Kops GJPL, Lam EWF, Burgering BMT, and Medema RH: Cell cycle inhibition by FoxO forkhead transcription factors involves downregulation of cyclin D. Mol Cell Biol 2002, 22:7842-7852

40. Hoogeboom D, Essers MAG, Polderman PE, Voets E, Smits LMM, Burgering BMT: Interaction of FOXO with + -catenin inhibits + catenin/T cell factor activity. J Biol Chem 2008, 283:9224-9230

41. Ding W, Bellusci S, Shi W, Warburton D: Functional analysis of the human Sprouty2 gene promoter. Gene 2003, 322:175-185

42. Brown MD, Kelsall CJ, Milkiewicz M, Anderson S, Hudlicka O: A new model of peripheral arterial disease: sustained impairment of nutritive microcirculation and its recovery by chronic electrical stimulation. Microcirculation 2005, 12:373-381

43. Asada S, Daitoku H, Matsuzaki H, Saito T, Sudo T, Mukai H, Iwashita S, Kako K, Kishi T, Kasuya Y, Fukamizu A: Mitogen-activated protein kinases. Erk and p38, phosphorylate and regulate Fox01. Cell Signal 2007, 19:519-527

44. Brenkman AB, de Keizer PLJ, van den Broek NJF, Jochemsen AG, Burgering BM: Mdm2 induces mono-ubiquitination of FOXO4. PLoS ONE 2008, 3:e2819 